\title{
The Transurethral Suprapubic endo-Cystostomy (T-SPeC): A Novel Suprapubic Catheter Insertion Device
}

\author{
Lawrence I. Karsh, MD, R. Blair Egerdie, MD, ${ }^{2}$ David M. Albala, MD, ${ }^{3}$ and Brian J. Flynn, MD ${ }^{4}$
}

\begin{abstract}
Background and Purpose: Current methods of suprapubic cystostomy (SPC) catheter insertion may be difficult for patients in poor health and can result in significant morbidity and mortality. These include a highly invasive open procedure, as well as the use of the percutaneous trocar punch methods, commonly associated with short-term SPC. We present the first human experience with the Transurethral Suprapubic endo-Cystostomy (T$\mathrm{SPeC}^{\circledR}$ ) device, a novel disposable device used for introducing a suprapubic catheter via a retrourethral (insideto-out) approach similar to the Lowsley technique.

Patients and Methods: Four men at St. Mary's General Hospital in Kitchener Ontario, Canada, received the TSPeC device (model T7) under general anesthesia.

Results: Patients had no complications from catheterization using the T-SPeC T7 Surgical System. The mean surgical time of the four procedures was 9.7 minutes, with a range of 7.9 to 13.5 minutes, including instrument preparation and cystoscopy. All four procedures were highly accurate and rapid. There were no complications and minimal blood loss from the procedure.

Conclusions: We found that the T-SPeC device allows for efficient and safe insertion of a suprapubic catheter in an outpatient setting and may be a useful addition to the urologic armamentarium. The T-SPeC Surgical System facilitates rapid and precise suprapubic catheter placement.
\end{abstract}

\section{Introduction}

$\mathbf{P}$ ATIENTS NEEDING PERMANENT CATHETERIZATION or longterm, repeated catheter insertion pose challenges to urologic management. Although the complications of chronic urethral catheterization, including urethral erosion, penile/ meatal discomfort, strictures, infection, and hygiene issues, are well-known, there is an understandable reluctance to submit these patients to an open operation. The patients are often fragile, in poor health, and at high risk for intra- and post-operative complications. If a suprapubic catheter is placed using an open technique, it will necessitate an operating room, anesthesia, and a postoperative recovery room for a few hours, even if the surgery is uncomplicated, placing additional costs on health care systems.

Suprapubic cystostomy (SPC) can currently be performed by percutaneous methods (outside-to-in); however, the accepted standard has been the open approach under general or spinal anesthetic. In 1988, Zeidman and colleagues ${ }^{1}$ described a technique using the Lowsley retractor originally designed for perineal prostatectomy. They reported a low complication rate, which was recently corroborated among patients with neurogenic bladder by Edokpolo and Foster. ${ }^{2}$ Although the Lowsley technique, using a reusable sterilized device, is effective, it has its limitations. The device is not readily available in many institutions, because it has been taken off the market and only used instruments are currently available. It necessitates a small abdominal incision and an assistant to provide retraction.

The Lowsley retractor was not specifically designed for SPC catheter insertion; hence, catheter engagement is not always guaranteed. As such, the catheter can become detached from the device at a critical moment when pulling the catheter through the fascia and bladder wall into the bladder lumen. This results in a failed placement and necessitates a second attempt at catheter placement and a second cystotomy. In addition, obese patients pose a technical challenge because the pannus may exceed the length of the instrument necessitating a cut-down incision to locate and deliver the device.

The Transurethral Suprapubic endo-Cystostomy (T$\mathrm{SPeC}^{\circledR}$ ) Surgical System is a novel disposable device used for introducing a suprapubic catheter via a retrourethral approach (inside-to-out) similar to the Lowsley technique. Similar to the Lowsley technique, the T-SPeC uses a curved

\footnotetext{
${ }^{1}$ The Urology Center of Colorado, Denver, Colorado.

${ }^{2}$ Urology Associates/Urologic Medical Research, Kitchener, Ontario, Canada.

${ }^{3}$ Associated Medical Professionals, Syracuse, New York.

${ }^{4}$ University of Colorado Denver, Denver, Colorado.
} 
stainless steel sound for proper location within the bladder, close to the pubic bone. Unlike the Lowsley technique, the T$\mathrm{SPeC}$ instrument initiates a $15 \mathrm{~F}$ surgical tract from within the bladder, starting at the targeted location, and exiting the abdomen, instead of cutting down to the tip of the sound with the Lowsely device. It allows for the precise placement of an 18 F silicone catheter with Councill tip without the need for an open cystostomy.

The suprapubic catheter placement is initiated in the inside of the bladder after the sound guidance system is placed transurethrally. Placement using the T-SPeC device obviates the need for fluoroscopy, ultrasonography, or guidewires and is performed as an outpatient procedure under general anesthesia, regional anesthesia, or even a combination of local anesthesia with minimal sedation. Before regulatory clearance, cadaveric studies were conducted; here, we present our initial human experience with the T-SPeC device in four live patients.

\section{Patients and Methods}

\section{Patients}

Four patients were identified for SPC - three with neurogenic bladders and one with postradiation urinary retention. Patients were excluded from the study if they had evidence of urethral stricture.

All four patients and their families were informed that they would be the first patients to experience this technique. Informed consent was obtained for all patients. The clinical procedures were performed at St. Mary's General Hospital in Kitchener, Ontario, Canada, on August 15, 2012.

\section{T-SPeC Surgical System}

The T-SPeC Surgical System (Fig. 1) (Swan Valley Medical Inc., Bigfork, MT) was licensed for use in Canada as of March 26, 2012. All four patients received the T7 model, which is designed for use with patients with a surgical tract of $7 \mathrm{~cm}$ or less. There is also a T14 model, allowing for a surgical tract depth of $14 \mathrm{~cm}$ or less.

Surgical technique. The procedures were performed under general anesthesia on an outpatient basis. After induction, a flexible cystoscopic examination was performed to ensure that there was no urethral pathology that would preclude the introduction of the T-SPeC sound. The sound was lubricated and gently inserted along the urethra and advanced into the bladder (Fig. 2A). The sound was then angled upward, toward the abdominal wall, and positioned to palpate the sound below the skin cephalad to the pubis (Fig. 2B). Palpation is not needed for proper placement and, in fact, cannot be accomplished in the obese patient, because of pannus size. The positioning arm and blade capture housing was then brought down along the mast indicating the exit position of the surgical blade on the skin (Fig. 2C). The device is designed with precise alignment between the tip of the sound and the blade capture housing, allowing accurate determination of the surgical tract. By sliding the sound handle forward, a blade attached to a coaxial coil was advanced from the tip of the sound upward, through the abdominal wall, until it engaged the blade capture housing (Fig. 2D). The blade capture housing, containing the blade, was then removed (Fig. 2E), and the catheter was then attached to the bayonet connector on the coaxial coil above the skin surface (Fig. 2F-G).

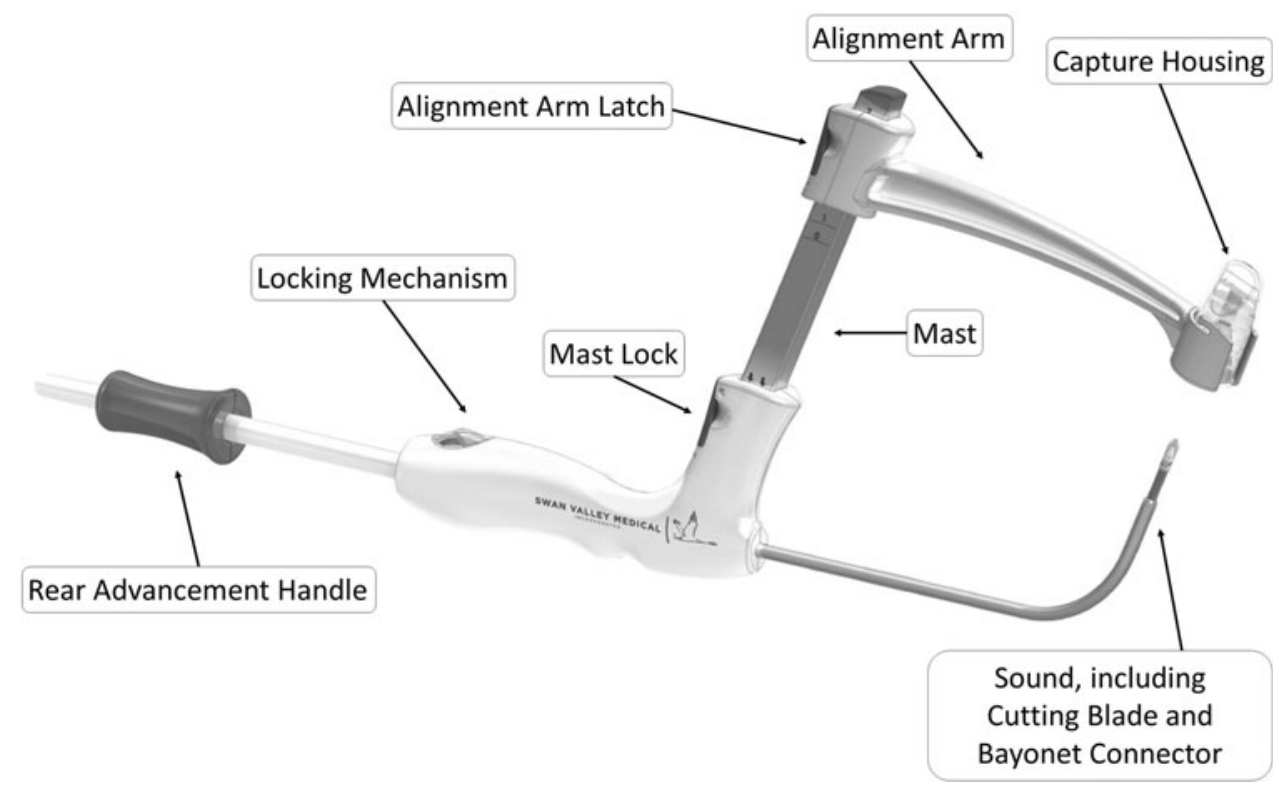

FIG. 1. Schematic of the T-SPeC Surgical System T7 device. The rear advancement handle advances the cutting blade (15F) from inside the bladder, through the bladder wall and abdomen, and pulls the catheter connected to the bayonet connector on the coaxial coil back through the surgical pathway for placement in the bladder. The locking mechanism in the rear handle locks the advancement handle, which controls the blade advancement. The mast guides the alignment guide arm down to the patient's abdomen before the creation of the surgical pathway. Abdominal thickness can be measured using the graduated mast. The positioning arm provides the surgeon with the blade exit point. The cutting blade makes a small incision (15F) through the bladder wall, fascia, and abdomen. It is housed within the sound and is deployed by the rear advancement handle. The capture housing accepts the surgical blade once it has passed through the patient's bladder wall and abdomen. The blade and capture housing can be removed for disposal by twisting the housing. 
FIG. 2. Surgical technique for the T-SPeC Surgical System. Insertion of the lubricated sound through the urethra (A). Angling of the sound toward the abdominal wall (B). Alignment of the positioning arm and blade capture housing (C). Advancement of the blade attached to a coaxial coil from the tip of the sound through the abdominal wall (D). Removal of the blade capture housing (E-F). Attachment of the catheter to the bayonet connector on the coaxial coil above the skin surface (G). The catheter visible at the meatus (H). Disengagement of the catheter by transection with scissors just above the bayonet connector (I). Visualization of the catheter tip in the bladder ( $\mathrm{J})$. Inflation of the catheter balloon with $10 \mathrm{~mL}$ of sterile water and cystoscopic confirmation of the balloon at the dome of the bladder $(\mathbf{K}-\mathbf{L})$. Anchoring of the catheter to the skin using a 3-0 nylon stitch (M).
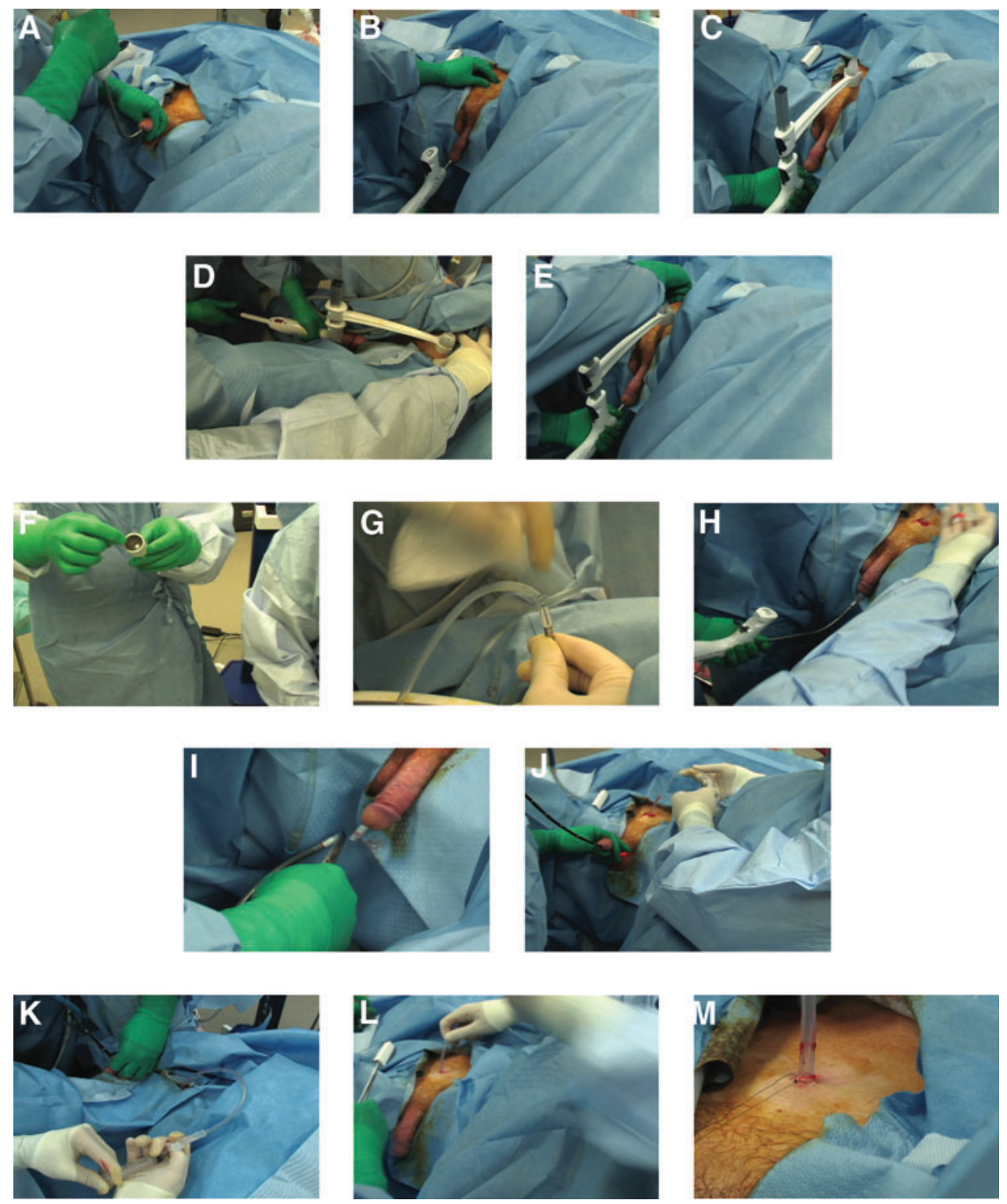

A $5 \mathrm{~mm}$ knick in the skin was made next to the sound to enlarge the skin opening to $18 \mathrm{~F}$. The sound, with the attached catheter, was then withdrawn in an antegrade fashion through the urethra until the catheter was visible at the meatus (Fig. 2H). The catheter was then disengaged by dividing it with scissors just above the bayonet connector (Fig. 2I). The catheter was then slowly drawn back into the bladder under direct flexible cystoscopic guidance until the tip was visualized in the bladder (Fig. 2J). The catheter balloon was inflated with $10 \mathrm{~mL}$ of sterile water. The position of the balloon at the dome of the bladder was confirmed cystoscopically (Figs. 2KL). The catheter was then anchored to the skin with a 3-0 nylon stitch (Fig. 2M). After anesthesia was reversed, each patient was transferred to the postanesthesia care unit and, after full recovery, was discharged from the hospital in good condition.

\section{Results}

Patient characteristics are summarized in Table 1 . Three of the patients had underlying neurologic conditions necessi- tating the placement of a permanent suprapubic catheter, while the fourth patient needed chronic catheterization after radiation therapy for prostate cancer. This patient elected for suprapubic tube placement because he experienced intolerable discomfort with the urethral catheter and refused to do clean intermittent catheterization. Two of the three patients with neurologic conditions had meatal urethral erosions extending to the midshaft area. All four patients had a body mass index (BMI) below 27. The TSPeC T7 device was chosen for use with the patients' narrow abdominal thicknesses. The TSPeC T14 is available for patients who have large abdominal thicknesses and generally have a BMI greater than 27. The difference between the T7 and T14 models is a longer coaxial coil in the T14 device to accommodate an obese patient with a larger pannus.

Figure 3 demonstrates the mean time for the four measured procedure phases: Cytoscopic bladder review (1.6 min; range: $0.9-2.9 \mathrm{~min}$ ); tray open/assemble (1.1 min; range: $0.9-$ $1.3 \mathrm{~min}$ ); surgical procedure (6.0 $\mathrm{min}$; range: $4.8-8.4 \mathrm{~min})$; and suture (1.0 $\mathrm{min}$; range: $0.8-1.2 \mathrm{~min})$. The mean total time was 
Table 1. Patient Characteristics

\begin{tabular}{|c|c|c|c|}
\hline $\begin{array}{l}\text { Patient } \\
\text { number }\end{array}$ & Age & Sex & Characteristics \\
\hline 1 & 72 & Male & $\begin{array}{l}\text { Paraplegic after a fall } \\
\text { Chronic catheter } \\
\text { Recent bladder stone removed }\end{array}$ \\
\hline 2 & 45 & Male & $\begin{array}{l}\text { Multiple sclerosis } \\
\text { Seizures } \\
\text { History of bladder and } \\
\text { kidney stones } \\
\text { Urosepsis } \\
\text { Chronic catheter }\end{array}$ \\
\hline 3 & 59 & Male & $\begin{array}{l}\text { Cerebrovascular accident } \\
\quad \text { (three strokes) } \\
\text { Diabetes } \\
\text { Chronic catheter }\end{array}$ \\
\hline 4 & 75 & Male & $\begin{array}{l}\text { Prostate cancer } \\
\text { Radiation treatment } \\
\text { Chronic urinary retention }\end{array}$ \\
\hline
\end{tabular}

9.7 minutes with a range of 7.9 to 13.5 minutes. The total time does not include the time between procedure steps (eg, walking from prep table to patient, preparing scope) or preand postprocedure debriefing. All procedures accurately placed the catheter with only one attempt at blade advancement (Fig. 3) and no episodes of intraoperative or postoperative catheter dislodgment.

There were no complications such as urine leak, bowel injury, wound infection, or hemorrhage. The patients were discharged the same day and returned to the clinic for catheter exchange in 6 weeks. As of October 21, 2012, all four patients have had the suprapubic tube changed without any difficulty or complications.

\section{Discussion}

Currently, clinicians use three common methods for placing a suprapubic catheter: (1) Open abdominal approach, also known as an open approach; (2) open abdominal placement of the suprapubic catheter using the Lowsley retractor device; and (3) percutaneous or punch suprapubic technique (outside-to-in).

The open abdominal approach is considered by many to be the gold standard for suprapubic catheter placement. The method necessitates a surgical incision to expose the bladder, requiring in most cases regional or general anesthesia, and the catheter is then placed under direct vision. The advantage of this method is that it is safe and reliable, especially in patients with previous abdominal surgery, radiation, adhesions, or a small contracted neurogenic bladder. ${ }^{3}$ This technique is useful for properly placing the catheter and providing reliable drainage. The primary disadvantages to the open abdominal approach are the invasiveness and cost.

Open abdominal placement of the suprapubic catheter using the Lowsley retractor device necessitates a smaller incision, less anesthesia, and less operative time compared with the open approach. ${ }^{1}$ Edokpolo and Foster ${ }^{2}$ reported their experiences using the Lowsley retractor method for suprapubic catheterization in 44 patients with neurogenic bladder. While they included female patients, their patients had similar demographic characteristics compared with the four patients in this study. They reported minimal blood loss and no intraoperative complications or incorrect catheter placements. The majority of their patients received general anesthesia. Notably, the mean operative time documented was $20.2 \pm 5.5$ minutes, with a range of 11 to 31 minutes. $^{2}$ Edokpolo and Foster, however, do not report how the surgical time was measured, so direct comparisons between the two systems cannot be made.

The Lowsley retractor method technique may not be appropriate in patients with surgical adhesions, because there is

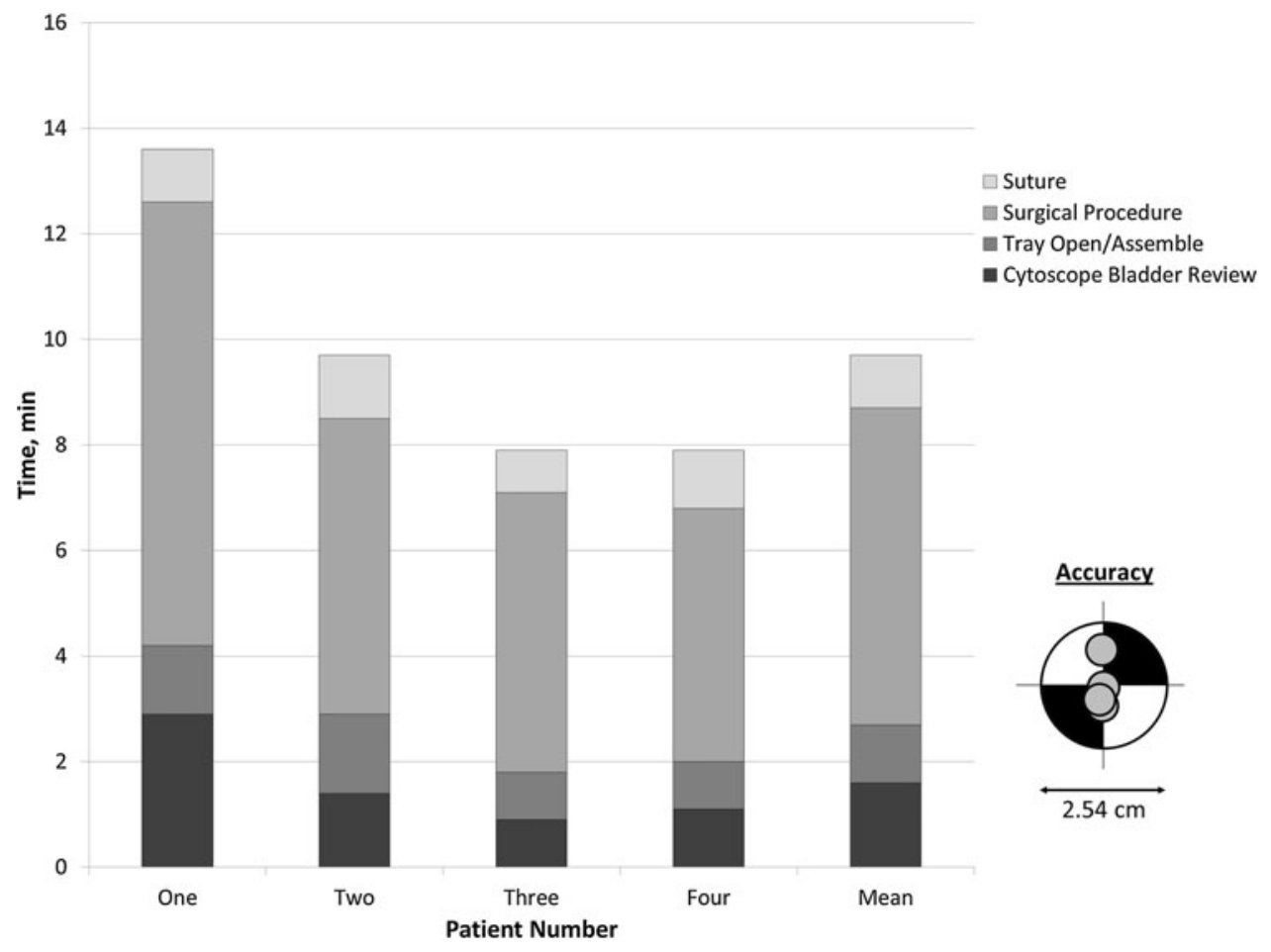

FIG. 3. T-SPeC Surgical System procedure time and accuracy. Procedure times for the four patients, as well as the mean time, are shown. The procedure time is broken down into cystoscopic bladder review, tray open/assemble, surgical procedure, and suture. Accuracy for the four procedures is shown in the lower right corner. 
potential for bowel injury. ${ }^{3}$ Also, because the Lowsley retractor may not reach the skin in obese patients, a larger incision may be needed for these patients to place retractors. The Lowsley retractor does not consistently grab the catheter tip, which may result in catheter dislodgement when pulling through the abdominal wall, necessitating a second cystotomy.

The percutaneous or punch suprapubic technique (outside-to-in) was used initially in patients with urethral stricture disease. The technique has gained wide popularity, because an incision is not needed and it can be performed in a nonoperating room setting such as the clinic or emergency room. Percutaneous suprapubic catheterization needs minimal or no sedation and convalescence. ${ }^{4}$ Therefore, percutaneous suprapubic catheterization is now used commonly in patients with normal urethras to serve as a temporary suprapubic catheter after pelvic surgery, and as a permanent catheter in patients with neurogenic bladder. ${ }^{5}$ It is the preferred method for many physicians for suprapubic catheter placement (Swan Valley Medical internal correspondence, data not shown).

Similar to the Lowsley retractor technique, it is not considered appropriate in patients with surgical adhesions, because there is potential for bowel injury. Another concern is the potential for misplaced suprapubic catheters, which can lead to perforation of the peritoneum cavity, puncture of the small bowel, and nonfunctional catheter. ${ }^{6-9}$ Unlike the open approach or Lowsley retractor technique, there is a limit on size of the suprapubic catheter $(10-14 \mathrm{~F})$ when placed with a percutaneous kit. ${ }^{4}$ A smaller caliber has the potential to become occluded with debris, blood, or mucus, resulting in unreliable urinary drainage for patients needing long-term drainage. In obese patients, the percutaneous kit may not reach or be able to locate the bladder. ${ }^{4}$ In a larger retrospective study, Ahluwalia and coworkers ${ }^{10}$ investigated 219 patients who underwent percutaneous suprapubic insertion under cystoscopic guidance, finding a 10\% intraoperative complication rate, including a $2.5 \%$ risk of bowel injury, a 30 -day complications rate of $19 \%$, and a mortality rate of $1.8 \%$.

Despite these risks, many patients prefer suprapubic catheter placement to transurethral catheters, arguing for the need for improved suprapubic catheterization techniques. ${ }^{7}$ The T-SPeC Surgical System was therefore developed in response to the need for a safe, easy, and effective procedure. As a first step, the effectiveness of the novel cystostomy system was studied in 14 human cadavers. Male and female cadavers were used with a BMI range of 28 to 43 . In all cases, successful suprapubic catheter placement was achieved. These studies led to the approval of the T-SPeC Surgical System for human use.

We found the T-SPeC Surgical System to be safe and effective in these first four patients. In our first 3 months of follow-up, there have been no significant complications such as catheter dislodgement, hemorrhage, or bowel injury. After only four patients in this study, the operative time averaged 9.7 minutes. Furthermore, while general anesthesia was used on all four of our patients, we believe that this technique can be performed using local anesthesia and no-to-minimal sedation in selected patients in the clinic setting. We do not anticipate the T-SPeC Surgical System to be a viable procedure in an emergency setting, however, such as at the bedside in an inpatient or in the emergency department, because the large majority of these patients have a compromised urethra that prohibited Foley catheter placement.

In addition, the catheter in the T-SPeC surgical kit has an open tip, equivalent to a Councill-tip catheter after it is transected at the urethra meatus. This feature allows for the insertion of a guidewire if necessary at the time of catheter change, especially if a larger catheter is needed at a later date. The primary disadvantage of the T-SPeC device-like the Lowsley retractor method-is that a patent urethra is needed. Also, using the T-SPeC device may be considered contraindicated in patients with surgical adhesions, because there is still a potential for bowel injury. We think that this risk is much lower than percutaneous techniques but not as safe as an open approach. We believe the unique design of the T-SPeC device will allow safe suprapubic catheter placement in many patients with abdominal adhesions, but this will need further study before this can be recommended.

Although our first four patients were men, this technique is not limited to male patients. Indeed, we anticipate that the procedure may be even easier, and faster, in women. In addition, the available T-SPeC T14 facilitates suprapubic catheter placement for large and very large patients. This model is designed to accurately create a surgical tract up to a $14 \mathrm{~cm}$ abdominal thickness, as commonly seen in obese and even morbidly obese patients. Current methods to place a suprapubic catheter in patients with a BMI over 27 are extremely difficult and provide additional risk by attempting to puncture the bladder with a needle from outside-to-in. We plan to report our experience with both female and obese patients in the near future.

\section{Conclusion}

The T-SPeC device allows a novel, simple, and accurate method of placing a suprapubic catheter with an inside-to-out approach. It allows for efficient and safe insertion of a longterm suprapubic catheter in an outpatient setting and should be a useful addition to the urologic armamentarium. We believe that the T-SPeC Surgical System will facilitate a more rapid and precise suprapubic catheter placement than open or outside-to-in percutaneous approaches.

\section{Acknowledgments}

T-SPeC devices were provided by Swan Valley Medical Incorporated. We would like to thank Tim Braun and Ronald Zook of Swan Valley Medical Incorporated for help in preparing and reviewing this manuscript. Medical editor Michael Linde of Linde Medical Writing, LLC, assisted in the preparation of this manuscript.

\section{Disclosure Statement}

Drs. Karsh and Albala are shareholders and advisory board members; Dr. Egerdie is a shareholder; and Dr. Flynn is a consultant and investigator for Swan Valley Medical, Incorporated.

\section{References}

1. Zeidman EJ, Chiang H, Alarcon A, Raz S. Suprapubic cystostomy using Lowsley retractor. Urology 1988;32:54-55.

2. Edokpolo LU, Foster HE Jr. Suprapubic cystostomy for neurogenic bladder using Lowsley retractor method: A procedure revisited. Urology 2011;78:1196-1198. 
3. Harrison SC, Lawrence WT, Morley R, et al. British Association of Urological Surgeons' suprapubic catheter practice guidelines. BJU Int 2011;107:77-85.

4. Irby PB, 3rd, Stoller ML. Percutaneous suprapubic cystostomy. J Endourol 1993;7:125-130.

5. Krane LS, Bhandari M, Peabody JO, Menon M. Impact of percutaneous suprapubic tube drainage on patient discomfort after radical prostatectomy. Eur Urol 2009;56:325-330.

6. Ahmed SJ, Mehta A, Rimington P. Delayed bowel perforation following suprapubic catheter insertion. BMC Urol 2004;4:16.

7. Ananthakrishnan K, Ayyathurai R, Chiran JK, et al. An unusual complication of suprapubic catheter insertion. ScientificWorldJournal 2006;6:2433-2435.

8. Barai KP, Islam S. Suprapubic catheterization complicated by an iatrogenic enterocutaneous fistula: A case report. Cases J 2009;2:9311.

9. Mongiu AK, Helfand BT, Kielb SJ. Small bowel perforation during suprapubic tube exchange. Can J Urol 2009;16:4519-4521.
10. Ahluwalia RS, Johal N, Kouriefs C, et al. The surgical risk of suprapubic catheter insertion and long-term sequelae. Ann R Coll Surg Engl 2006;88:210-213.

Address correspondence to: Lawrence I. Karsh, MD The Urology Center of Colorado 2777 Mile High Stadium Circle Denver, CO 80204

E-mail: larrykarsh@gmail.com

$\begin{aligned} & \text { Abbreviations Used } \\ & \mathrm{BMI}=\text { body mass index } \\ & \mathrm{SPC}=\text { suprapubic cystostomy } \\ & \mathrm{T}-\mathrm{SPeC}=\text { Transurethral Suprapubic endo-Cystostomy }\end{aligned}$

